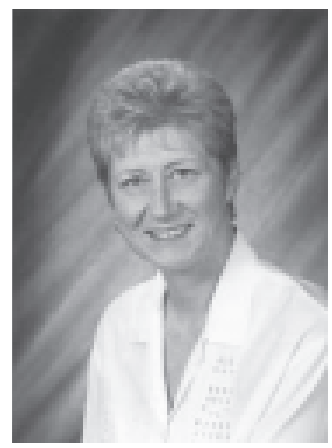

\title{
Linda und Mark Sobell: Problemverhalten statt Sucht? Selbsthilfe statt Therapie?
}

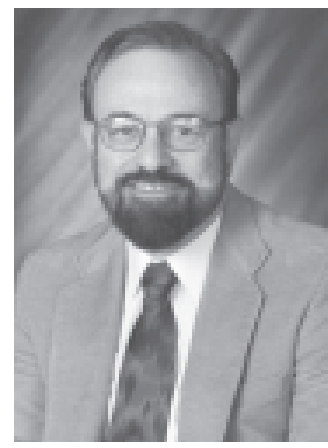

Prof. Dr. Linda und Prof. Dr. Mark Sobell sind - nach früheren Professuren in Toronto und an der Addiction Research Foundation in Kanada - gegenwärtig Professoren für klinische Psychologie an der Nova Southeastern University in Florida. National und international sind sie bekannt geworden durch ihre klinischen Forschungen im Suchtbereich, besonders zu kurzen, motivationalen Interventionen und zur Förderung von Spontanremissionen bzw. Selbstveränderung. Linda Sobell hat Wissenschaftspreise der Amerikanischen Psychologischen Gesellschaft, der Association for the Advancement of Behavior Therapy und der Harvard Universität erhalten. Sie ist Past-President der Association for the Advancement of Behavior Therapy und President Elect der Society of Clinical Psychology der Amerikanischen Psychologischen Gesellschaft. Mark Sobell ist Acting Editor des Journal of Consulting and Clinical Psychology. Für seine Arbeiten erhielt er unter anderem einen Wissenschaftspreis der Amerikanischen Psychologischen Gesellschaft und den Jellinek Memorial Award. Für massive Unruhe in der Suchtforschungs- und -therapieszene sorgten Publikationen über kontrolliertes Trinken Anfang der 1970er Jahre. Das Interview führte Prof. Dr. Iver Hand, Hamburg, via E-mail.

Als klinische Psychologen haben Sie Ihr berufliches Leben einem ungewöhnlichen Schwerpunkt gewidmet: Der Propagierung von Selbstveränderung. Was hat Sie dazu veranlasst?

Linda und Mark Sobell: Nun, wir haben unser Leben nicht der Propagierung von Selbstveränderung gewidmet. Das war ein Entwicklungsprozess, der sich aus unserer ursprünglichen Beschäftigung mit klinischen Studien in einer Therapieeinrichtung ergab. Unsere erste Studie befasste sich mit individualisierter Verhaltenstherapie. Daraus resultierten weitere klinische Studien. Irgendwann in den späten 1970er Jahren stießen wir dann auf die Arbeiten von George Vaillant und Barry Tuchfeld, die nahe legten, dass Spontanremission oder Selbsthilfe möglich sind. Damals war nicht klar, wie viele Personen ohne fachliche Hilfe oder Therapie genesen können. Ab den 80er Jahren haben wir dann das Phänomen der Spontanremission oder Selbsthilfe untersucht. Dabei arbeiteten wir unter anderem mit Dr. Harald Klingemann zusammen. Bald wurde durch mehrere kanadische Studien deutlich, dass spontane Remissionen von Alkohol- und anderen Drogenproblemen häufig sind. Typischerweise passiert dies bei Personen, deren Drogenprobleme nicht so schwer sind, wie bei Personen, die in Therapie gehen. So hatten wir in den frühen 90er Jahren gute Gründe, auf Menschen zuzugehen, die trotz Alkohol- oder Drogenproblemen keine therapeutische Hilfe aufsuchten. Dies ist die Mehrheit. Die entscheidende Frage war, wie wir Selbsthilfe für diese Menschen attraktiv ma- chen könnten. Wir gingen davon aus, dass frühe Interventionen sich für die Betroffenen wie für die Gesellschaft deutlich Kosten senkend auswirken. Aus unseren Studien über Spontanremission entwickelten wir empirisch begründete Motivationsstrategien, die zu unserer allerjüngsten Studie führten.

Ihr Buch aus dem Jahr 2001 hat den Titel «Promoting Self-Change from Problem Substance Use». Wie steht es mit den Möglichkeiten zur Selbsthilfe bei Substanzmissbrauch oder -sucht?

Linda und Mark Sobell: Das ist eine gute Frage. Wir haben uns wohl zufällig für das Wort «use», Gebrauch, im Titel entschieden, aber in der Tat ist es sinnvoll, Selbsthilfe für praktisch jede Form des Suchtverhaltens zu propagieren.

Sie haben in der professionellen Suchttherapieszene in den frühen 1970er Jahren mit Ihren Feststellungen, dass manche Personen mit Suchtproblemen durchaus zu Normalverhalten, z.B. sozialem Alkoholtrinken, zurückkehren können, ohne später wieder in süchtiges Alkoholtrinken zu verfallen, eine Revolution ausgelöst. In einer hässlichen ideologischen Kampagne wurde Ihnen sogar Datenbetrug vorgeworfen, und Sie gingen mehrere Jahre in die «wissenschaftliche Emigration» nach Kanada. Warum ist dies geschehen und was veranlasste Sie, in Ihr Heimatland zurück zukehren?

\begin{tabular}{ll}
\hline KARGER & ( 2004 S. Karger GmbH, Freiburg \\
$\begin{array}{ll}\text { Fax +497614520714 } & \text { Accessible online at: } \\
\text { E-mail Information@Karger.de } \\
\text { www.karger.com }\end{array}$ & www.karger.com/ver \\
&
\end{tabular}

Linda C. Sobell, Ph.D. / Mark B. Sobell, Ph.D 
Linda und Mark Sobell: Sie sind nicht der Erste, der meint, dass wir aus den USA verjagt wurden, aber das ist vollkommen falsch. Unsere Studie über kontrolliertes Trinken wurde zwischen 1970-1972 durchgeführt. Wir haben von 1974-1980 an der Vanderbilt University gelehrt und wurden dort von der angesehenen Addiction Research Foundation angeworben, wo wir bis 1996 gearbeitet haben. Von dem Angriff auf unsere frühe Forschung erfuhren wir erst im Februar 1982. Wir waren von der Addiction Research Foundation bereits eineinhalb Jahre vor dieser Attacke angeworben worden und nicht gezwungen, in ein anderes Land auszuwandern. Warum wir zurückkehrten, ist eine interessante Frage. Wir haben nie nach neuen Arbeitsplätzen gesucht. Wir wurden immer von den alten Arbeitsplätzen abgeworben, ob dies nun die Vanderbilt University, die Addiction Research Foundation oder unser jetziger Arbeitsplatz, die Nova Southeastern University, war. Wir waren sehr, sehr glücklich über die Möglichkeit, unsere Kinder in Kanada aufziehen und dort arbeiten zu können. Aber das Arbeitsplatzangebot in Florida war extrem gut, so dass wir uns entschieden, in die USA zurückzukehren. Keiner unserer Arbeitsplätze hatte jemals etwas mit der Kontroverse über kontrolliertes Trinken zu tun.

$\mathrm{Zu}$ dieser Kontroverse kam es vermutlich, weil der Alkoholbereich damals, wie Thomas Kuhn wohl sagen würde, in einem prä-paradigmatischen Zustand war; der Alkoholbereich als Forschungsfeld war lange Zeit in einem vorwissenschaftlichen Stadium geblieben. Anfang der 70er Jahre begannen sich dann mehrere Dinge in Nordamerika zu verändern: das National Institute of Alcoholism and Alcohol Abuse und andere staatliche Einrichtungen wurden aufgebaut und sollten die Therapie und die Forschung bei Alkoholproblemen vorantreiben. Forschungsgelder wurden nur an promovierte Psychologen oder Ärzte mit einem Forschungshintergrund vergeben. Aus dieser Entwicklung entstanden nach und nach Forschungsstudien und Publikationen, die zu tiefer gehenden Veränderungen im Alkoholbereich führten. Viele empirische Ergebnisse widersprachen den damals gängigen naiven Suchtvorstellungen und stellten die Genesungsregeln der abstinenten Alkoholiker, die in diesem Bereich als Therapeuten arbeiteten, in Frage.

Thomas Kuhn hat den Prozess des Paradigmenwechsels als eine wissenschaftliche Revolution bezeichnet, die zwangsläufig mit Auseinandersetzungen, Bedrohungen etc. einhergeht. In kleinerem Umfang hat das auch im Alkoholbereich stattgefunden, einschließlich des «Glaubenskrieges» mit den Anonymen Alkoholikern, die eine Hauptmacht in diesem Feld waren und sind. Die Auseinandersetzungen waren ja sehr leidenschaftlich und betrafen nicht nur unsere Forschungsergebnisse. Da war der Angriff auf Davies' Forschung, und die Rand Corporation wurde von den gleichen Personen attackiert, die auch uns angriffen. Als die Zahl der publizierten Studien über moderaten Stoffkonsum zunahm, war die Frage, wer am richtigen Platz zur falschen Zeit oder am falschen Platz zur richtigen Zeit war und unglücklicherweise waren wir das. Wichtig war, dass der Paradigmenwechsel so oder so kommen würde. Zum Glück für den Wissenschaftsbetrieb und für uns haben wir nicht mit falschen Daten gearbeitet und unsere Daten aufgehoben, so dass wir unsere Integrität und die der Studie verteidigen konnten.
Was hat sich in der professionellen Suchttherapie und -forschung verändert, seit Sie in die USA zurückgekehrt sind?

Linda und Mark Sobell: In den USA haben sich schon einige Dinge geändert. Die USA sind in vielerlei Hinsicht puritanisch, nicht nur in Bezug auf Süchte. Während Mäßigung und Harm Reduction in vielen Ländern (z.B. Kanada, Schweiz, Neuseeland, Australien, England) akzeptierte Ziele sind, ist dies in der amerikanischen Therapieszene noch nicht der Fall. In den USA wird das Konzept der Harm Reduction sehr kontrovers diskutiert.

Was hat sich also geändert? Es gibt viel mehr Studien zu LowRisk-Alkoholkonsum, aber vermutlich nicht aus wissenschaftlicher Neugierde, sondern eher weil nach heutiger Meinung eine gewisse Alkoholmenge, ein oder zwei Drinks einige Male pro Woche, positive Effekte, z.B. auf das Herz-Kreislauf-System, hat. Außerdem gibt es zwischenzeitlich Publikationen, die zeigen, dass hausärztliche Gespräche für die Einleitung und Aufrechterhaltung von Low-Risk-Alkoholkonsum hilfreich sein können. Ärzte sind heute stärker in die Behandlung von Problemtrinkern einbezogen als früher. In der Therapieszene wird jetzt auch akzeptiert, dass es ein Kontinuum von Alkoholproblemen gibt, so dass Studien mit Problemtrinkern für die Vertreter des Suchtkonzeptes wesentlich weniger bedrohlich sind als solche mit massiv abhängigen Personen. Insgesamt hat sich also einiges verändert - aber langsam, und in den USA sehr viel langsamer als in anderen Ländern. Jetzt werden ja auch in Deutschland verschiedene Untersuchungen über moderates Trinken publiziert; kontrolliertes Trinken kann ein Therapieziel auch in Deutschland sein [Koerkel J: Controlled drinking as a treatment goal in Germany. J Drug Issues 2002;32:667-688.].

In den USA ist das Suchtmodell von Durand Jacobs für stoffgebundene Süchte wie für pathologisches Glücksspielen sehr populär. Das gilt auch für die gegenwärtige Diskussion über Sucht als eine "Selbstmedikation», die unerträgliche negative Befindlichkeiten zu reduzieren hilft. Dieses und ähnliche Modelle haben wenig mit dem klassischen Suchtmodell gemeinsam, wie es von den Anonymen Alkoholikern und manchen professionellen Therapeuten vertreten wird. Als ich Durand Jacobs vor 2 Jahren auf einer Konferenz zu pathologischem Glücksspiel fragte, warum er seinen Ansatz als neues Suchtmodell bezeichne, antwortete er «andernfalls würde mir niemand im Suchtbereich zuhören». Wie interpretieren Sie das?

Linda und Mark Sobell: Nach unserer Erfahrung ist das Suchtmodell von Durand Jacobs in den USA nicht sehr bekannt. Tatsächlich war uns dieses Modell gar nicht bekannt, bis Sie Ihre Frage stellten, obwohl wir doch ganz gut darüber Bescheid wissen, welche Erklärungsmodelle für Sucht in den USA populär sind. Wir haben uns über dieses Modell kundig gemacht, und es stimmt, dass dieses und auch die Self-Medication-Modelle nahezu nichts gemeinsam haben mit dem klassischen Suchtmodell. In den USA ist nach wie vor das klassische Suchtmodell der 12 Schritte am populärsten, aber das am weitesten akzeptierte Alternativmodell ist wohl am ehesten ein kognitiv-verhaltens- 
orientierter motivationaler Ansatz. In der Forschung über die Ursachen steht ein multifaktorieller Ansatz im Vordergrund. Dieser überschneidet sich natürlich teilweise mit Jacobs' Modell, aber es gibt sehr viele Daten zu wesentlichen anderen Variablen, die unseres Wissens in Jacobs' Modell nicht berücksichtigt sind.

Sie haben geschrieben, dass die Begriffe «Alkoholiker» oder «Süchtiger» für Betroffene eine Stigmatisierung bedeutet, die diese davon abhält, in Therapie zu gehen, und keine klinischen Vorteile bringt. Sollten wir diese Begriffe aufgeben? Und wenn, für welche Alternative?

Linda und Mark Sobell: Durch die Darstellung in den Medien und auch die Veröffentlichung von Lebensgeschichten von Langzeitmitgliedern im 12-Schritte-Programm sind die Begriffe «Alkoholiker» und «Süchtige» zumindest in den USA sehr stigmatisierend geworden. Dies ist nicht verwunderlich, denn der Suchtbereich hat sich lange Zeit nur mit sehr schwer und chronisch Abhängigen am Rande der Gesellschaft befasst. Sie stellen für Menschen mit weniger schwer wiegendem Problemverhalten keine Identifizierungsmöglichkeit dar. Gleiches gilt für das lebenslange Etikett einer Normabweichung (label of deviance). Die gering Gestörten fühlen sich durch eine Stigmatisierung im Sinne der früheren stereotypen Ansichten über Süchtige bedroht. Außerdem ist die traditionelle Suchttherapie für sie unangemessen intensiv. Es gibt also viele Gründe, warum Problemtrinker traditionelle Suchttherapien vermeiden. Die Suchtforschung, soweit sie Glücksspielen mit umfasst, hat daher ein KontinuumKonzept von Suchtproblemen entwickelt - vom gering Abhängigen über den mittelstark bis hin zum massiv Abhängigen.

Unserer Meinung nach brauchen wir die Begriffe «Alkoholiker» und «Süchtige» nicht mehr. Sie haben keinerlei Nutzen für die Therapie. Im diagnostischen und statistischen Manual der Amerikanischen Psychiatrischen Gesellschaft, dem Hauptdiagnostikinstrument in den USA, werden diese Begriffe auch nicht benutzt. Sinnvoller scheint es, von Alkohol- oder Drogenproblemen zu sprechen. Viele Leute können zugeben, dass ihr Alkoholgenuss problematisch ist, aber sie wollen nicht als Alkoholiker oder Süchtiger bezeichnet werden.

Deshalb haben andere und wir in Broschüren, Handzetteln und ähnlichem Informationsmaterial eine andere Strategie entwickelt, um diese Menschen zu erreichen. In den Annoncen stellen wir Fragen wie: «Erwägen Sie, Ihr Trinkverhalten zu verändern?», «Haben Sie schon überlegt, ob Sie Ihr Trinkverhalten reduzieren wollen?». Solche Annoncen führen weltweit dazu, dass sich Problemtrinker in großer Zahl melden.

Können Sie den Anteil substanzabhängiger Süchtiger, denen Selbsthilfe geholfen hat, verlässlich schätzen?

Linda und Mark Sobell: In den bisherigen epidemiologischen Studien ist eine Trennung von Problemtrinkern und Personen mit massiven Alkoholproblemen nicht möglich. Die meisten epidemiologischen Untersuchungen legen nahe, dass $60-75 \%$ dieser Gesamtpopulation für $1 \mathrm{Jahr}$ oder länger ohne professionelle Hilfe oder Therapie gebessert waren.
Sie schreiben in Ihrem Buch mehrfach, dass viele Süchtige sich ohne Therapie bessern, ohne sich aber darüber im Klaren zu sein. Können Sie das erläutern?

Linda und Mark Sobell: Fast alle Personen, die ohne professionelle Hilfe oder Therapie eine Besserung erzielen, kommen nie in Kontakt mit Therapieprogrammen oder Therapeuten. Sie haben daher gar keine Gelegenheit, ihre Geschichten zu erzählen. Darüber hinaus gab es bis vor kurzem nur wenige Studien zur Spontanremission. Von daher war es schwierig wahrzunehmen, dass Selbsthilfe häufig erfolgreich ist.

Gibt es irgendwelche Daten dazu, wie viele frühere süchtige Alkoholiker oder Glücksspieler nach erfolgreicher Selbsthilfe oder Therapie einen Rückfall erleiden, wenn sie nach Eintritt des Erfolges soziale Trinker oder Glücksspieler werden?

Linda und Mark Sobell: Überzeugende Studien zu dieser Frage fehlen bisher. Eine kleinere Studie von uns legt nahe, dass Spontanremission und Therapieremission nach 3- bis 5-jähriger Stabilität nur noch eine geringe Rückfalltendenz beinhalten.

Wie bedeutend ist das Abstinenzgebot in der Suchttherapieszene im Jahr 2004 und gibt es unterschiedliche Meinungen zu stoffgebundenen und stoffungebundenen Süchten?

Linda und Mark Sobell: In den USA dominiert das Abstinenzgebot nach wie vor. Soweit wir die Situation in England, Australien und anderswo überblicken, besteht in diesen Ländern und in Kanada ein sehr viel rationalerer Ansatz in der Suchttherapie. Bezüglich der Therapieziele wird akzeptiert, dass es eine Indikation für Low-Risk-Drinking gibt, speziell bei Personen mit geringeren Alkoholproblemen. Bei schwerwiegenderen Alkoholproblemen oder bei anderen Drogenproblemen, ist aber Harm Reduction wahrscheinlich das angemessene Therapieziel. In den USA wird unter Harm Reduction üblicherweise verstanden, dass der Drogenkonsum reduziert wird. In England wird dieser Begriff eher für Maßnahmen benutzt, die die schädlichen Konsequenzen des Genusses reduzieren, wie der Zusatz von Vitaminen und Thiamin im Bier, um das Risiko einer Hirnschädigung zu reduzieren. In den USA würde demgegenüber die Reduktion des Konsums, aus der dann eine Reduktion des möglichen Schadens erwartet wird, als Harm Reduction bezeichnet werden.

Werden in den USA die Begriffe «Reduced Drinking» und «Harm Reduction» dann synonym gebraucht?

Linda und Mark Sobell: Ich glaube, ja - und dies anscheinend im Gegensatz zu Europa. Um es in Analogie zum Autofahren auszudrücken: In den USA wäre die Geschwindigkeitsbegrenzung Harm Reduction, in Europa wäre Harm Reduction die Erhöhung der aktiven und passiven Sicherheit der Autos.

Herzlichen Dank für das Gespräch! 